Graciela Fandiño Cubillos

Profesora Asistente Universidad Pedagógica Nacional

\title{
EL USO DEL JUGUETE EN LOS JARDINES INFANTILES
}

\section{PRESENTACIÓN}

Este trabajo se inscribe dentro de lo que se considera el paradigma del pensamiento del profesor cuyo supuesto principal considera que la actuación del profesor se encuentra en gran medida condicionada por su pensamiento y que este no es un reflejo objetivo y automático de la complejidad real. PÉREZ, GIMENO (1988) ${ }^{1}$, MARCELO $(1987)^{2}$. El estudio busca, entonces indagar las concepciones y prácticas de 4 maestras de preescolar en el uso del juguete en las actividades que realizan en el jardín, con el fin de determinar sus conceptos sobre juguete, sus usos y el papel que la maestra cumple en los usos de este. Lo anterior permite iniciar el conocimiento sobre el pensamiento de las maestras en esta temática.

La importancia del juego y el juguete en la vida del niño es tal vez una de las ideas más generalizadas en la educación infantil, ya que se considera que las conductas lúdicas son la esencia fundamental de la vida del niño y ocupa casi todo el tiempo del

\footnotetext{
${ }^{1}$ Pérez, Ángel y Gimeno, José. "Pensamiento y acción en el profesor: de los estudios sobre la planificación al pensamiento práctico", en Rev. Infancia y aprendizaje, vol. 42, Barcelona, 1988.

${ }^{2}$ García C., Marcelo. EI Pensamiento del profesor, CEAC, Barcelona, 1987.
}

niño. GLANZER (1992) $)^{3}$. Existe gran variedad de literatura sobre el tema publicados en libros, artículos, documentos oficiales, que argumentan y desarrollan este postulado. Así mismo la literatura sobre juguete se ha especializado en describir los buenos juguetes, sus características y ventajas de acuerdo a la edad del niño, pero no encontramos estudios que indaguen sobre los usos reales del juguete en los jardines infantiles y los porqués de estos usos según las maestras jardineras.

De acuerdo a lo anterior, al iniciar una exploración sobre el tema del juguete en el pensamiento de los maestros de preescolar, pretendemos plantear algunos interrogantes que den paso a nuevos investigaciones, así como aportar elementos de comprensión del pensamiento de las maestras que aporten a la capacitación que en este tema usualmente se realiza.

\section{EL MÉTODO}

El estudio es básicamente de naturaleza exploratoria-descriptiva y se efectúo a través de la me-

\footnotetext{
${ }^{3}$ Glanzer, Martha. "El rol estimulante de un buen juguete en el desarrollo del niño", en Un jardin de infantes mejor. Siete propuestas. Paidos, Buenos Aires, 1992.
} 
todología de estudio de caso. El análisis de la información se realizó con la técnica de análisis de contenido.

\section{Población}

Selección de los jardines y profesores interesados en participar en el estudio.

Con estos propósitos, y como preparación del trabajo posterior, en el primer semestre de 1998 se aplicó una encuesta introductoria a 22 profesoras de preescolar, quince de ellos alumnas del programa de profesionalización de la Universidad Pedagógica Nacional, las demás compañeras de trabajo de las mismas. La encuesta se limitó a dos preguntas:

1. ¿Qué juguetes utiliza en las actividades que usted realiza en el jardín?

2. ¿Para qué utiliza estos juguetes y con niños de qué edad?

La encuesta mostró que:

1. Los maestros dicen usar en su aula gran variedad de juguetes: muñecos, balones, carros y bloques de construcción son los más frecuentes.

2. Su utilización busca, principalmente, el desarrollo cognitivo, motriz, social y de la creatividad del niño.

A continuación se pasó a seleccionar cuatro jardines (que dieron muestras de colaboración y tenían menos problemas de lejanía), y se trabajó con las maestras que tuvieran los niños de mayor edad, ya que con estos niños se realizan actividades más

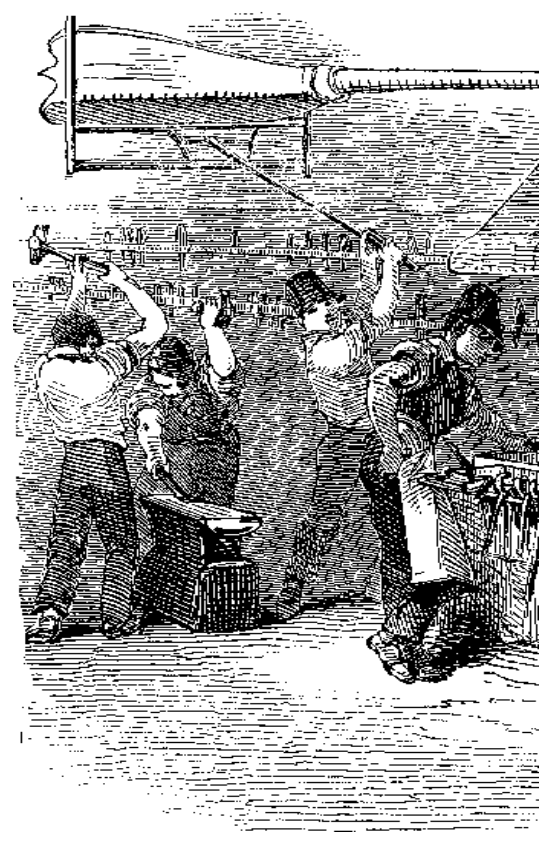

"escolarizadas" que buscan prepararlo para su futura escolarización y en este sentido el juego podría tener unos objetivos más definidos.

\section{Los jardines}

De los cuatro jardines donde se llevó a cabo el estudio, dos son privados y dos son de carácter oficial.

A los jardines privados asisten niños de estratos socioeconómicos medios altos, en su mayor parte hijos de profesionales y empleados, son casas familiares adapatadas como jardines infantiles, el número de niños que en total se atienden están entre 40 y 50 niños. En el primero se trabajó con 10 niños de 41/2 a 51/2 años de edad. En el segundo jardín se trabajó con 30 niños entre 21/2 y $41 / 2$ años, pues, la actividad fue organizada con todos los niños de esas edades.
Los jardines oficiales pertenecen al Instituto Colombiano de Bienestar Familiar (ICBF), entidad adscrita al Ministerio de Salud. Al primero acuden niños de estratos medios bajos y bajos. La maestra trabaja con 24 niños entre los $41 / 2$ y $51 / 2$ años de edad. El otro jardín es anexo a una institución oficial, acuden niños de estrato medio, principalmente hijos de trabajadores, de distintos rangos, de una entidad oficial. La maestra tiene 26 niños entre los $41 / 2$ y $51 / 2$ años de edad. Son espacios construidos como jardines infantiles y albergan entre 150 y 180 niños.

\section{Las maestras}

La maestra del primer jardín realizó la Licenciatura en Psicopedagogía y es su primer año de experiencia. La del segundo jardín es Licenciada en Educación Preescolar y tiene tres años de experiencia. La maestra del tercer jardín hizo una licenciatura en Administración Educativa y tiene diez años de experiencia. La del último jardín egresó hace ocho años de la Escuela Normal y cursa segundo semestre de la licenciatura en Educación Preescolar. Tiene ocho años de experiencia.

\section{Escenarios de trabajo}

Obtenido el consentimiento de instituciones y maestras para realizar con ellas el estudio, se les solicitó "organizar una actividad con juguetes". Se les explicó que dicha actividad sería filmada y se harían entrevistas. En los jardines objeto de observación se organizó la actividad en las horas de la mañana. 
La maestra del primer jardín llevó a los niños a un espacio abierto, (encima de la escalera) en donde se encontraban juguetes como cocinas, lavaplatos, etc. Además, llevó un canasto con más juguetes y los puso allí; luego les dijo a los niños: "van a jugar”. La actividad finalizó a los 30 minutos.

A un espacio, sin materiales, que está en comunicación con otros dos, fueron llevados los niños del segundo jardín. Se sentaron sobre una colchoneta y escucharon la lectura que la maestra les hizo de un cuento. Luego, la maestra les trajo una bolsa con juguetes que regó por el suelo. Los niños fueron hacia los juguetes. La actividad se terminó a los 35 minutos.

Los niños del tercer jardín jugaron en el salón que está asignado al grupo. En las partes altas de sus paredes hay repisas con

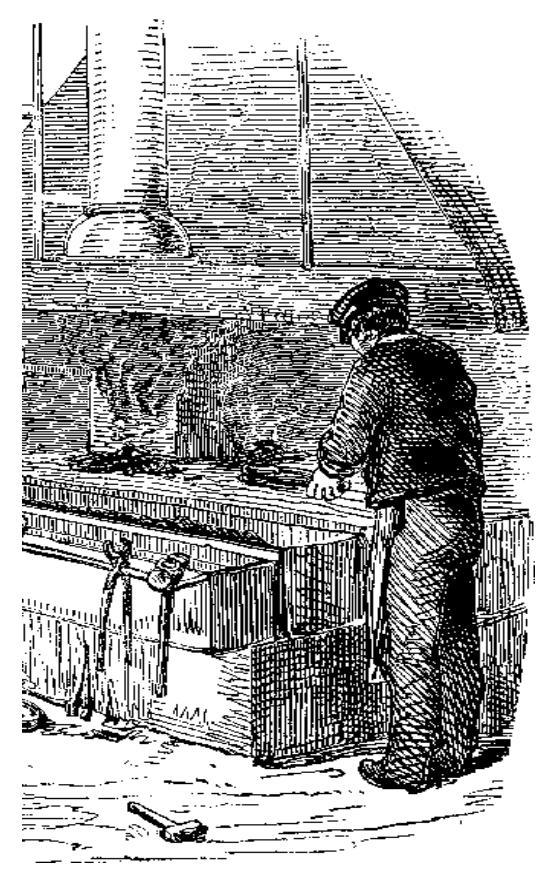

gran cantidad de juguetes, algunos todavía con sus empaques originales. Los niños se organizaron en círculo, sentados en sus sillas. La profesora les dijo que pensaran en el juguete que querían y les pidió que lo cogieran. Vueltos a sus sillas, la profesora pregunta a cada uno sobre el juguete que tomó. Ella les dice que jueguen. Pasados 20 minutos van al parque; luego de 30 minutos la actividad finaliza.

En el último jardín, la maestra organizó a los niños en grupos y les trajo dos bolsas con fichas plásticas: las repartió equitativamente y les pidió a los niños que hicieran con ellas lo que quisieran o lo que más les hubiera gustado del tema que habían trabajado antes (la casa sobre el agua). Al finalizar, los niños volvieron a su puesto y la profesora le pidió a cada uno que contara lo que había hecho. La actividad terminó a los 20 minutos.

\section{Procedimiento}

\section{Instrumentos}

Encuesta de sondeo, grabación en vídeo y entrevistas (las cuales fueron transcritas).

\section{Realización de la entrevista}

A las maestras se les solicitó "organizar una actividad con juguetes". Esta actividad fue filmada y durante la actividad y posterior a la actividad, cada maestra fue entrevistada. Una vez empiezan a jugar los niños, las maestras, desde la puerta o una esquina del salón observan a los niños, este momento es aprovechado para hacer parte de la entrevista.

\section{Análisis de la información}

El procesamiento de la información se realizó con la técnica de análisis de contenido. Los protocolos se elaboraron sobre las entrevistas, la filmación y las observaciones del jardín. Las dimensiones a analizar fueron: concepto de juguete, uso del juguete, función de la maestra en ese uso del juguete. Obtenidas las categorías, se procedió al análisis de la información, para sacar conclusiones.

La investigadora y dos colegas investigadores, procedieron de forma independientemente a leer los protocolos, identificando la información referente a cada una de las dimensiones objeto de indagación inicial. Luego se pusieron en común los resultados del ejercicio, donde se presentaron discrepancias se procuraron acuerdos o se acogió la opinión de la mayoría.

Cada investigador leyendo los textos identificados para cada dimensión, fue creando inductivamente categorías tentativas, atendiendo a rasgos de la información que permitiera agruparla por semejanzas semánticas ${ }^{4}$. En sesión colectiva se compararon y se fueron estableciendo los acuerdos acerca de las agrupaciones y su denominación.

\section{LOS RESULTADOS}

A las dimensiones que definieron el propósito inicial del presente

\footnotetext{
${ }^{4}$ Para esta labor fueron útiles las orientaciones de M. Martínez: La investigación cualitativa etnográfica en educación. Caracas: 1991. Capítulo V, Categorización y análisis.
} 
estudio -concepto de juguete, funciones que se le atribuyen y papel del maestro en su empleose agregaron dos más que emergieron en el desarrollo de la investigación: identificar y analizar los espacios y los tiempos de utilización del juguete. Dentro de todas las dimensiones surguieron las siguientes categorías inductivas:

\section{Concepto de juguete}

Las entrevistas realizadas indagan indirectamente por el concepto de juguete; tal vez por ello, sólo en un caso hay un concepto explícito de juguete: el juguete como objeto para jugar, "el juguete no tiene que ser algo específico sino que cualquier objeto puede ser juguete si hace parte del mismo juego".

Podemos afirmar que en los jardines investigados, donde se solicitó la realización de una actividad con juguete, éste es considerado como algo diferente al material didáctico, ya que disponiendo de gran variedad de material didáctico (rompecabezas de figura humana, letras, etc.) éste no fue usado, en tres jardines se usaron específicamente juguetes ya sea tomados del "canasto de los juguetes" o estantes y en uno se utilizó un armatodo de fichas grandes. Las actividades en los cuatro jardines, fueron de juego libre.

Si tenemos en cuenta, además, que no hubo intervención de los maestros sobre el juego en ninguna de las actividades, podría pensarse que el uso del juguete no implica una propuesta pedagógica en el sentido de que puede ser usado para una actividad intencional, con unos fines determinados.

\section{Funciones atribuidas al juguete}

Una aproximación al concepto de juguete la podemos hacer a partir de las funciones que se le atribuyen:

\section{El juguete como instrumento} de recuperación de actividades trabajadas en el aula.

Los maestros poseen intenciones y objetivos acerca de su trabajo con los niños. De ahí que una de las principales funciones que se le atribuyen al juguete sea la de continuar o recuperar las actividades estructuradas de aula, como son las de aprendizaje de conceptos, desarrollo motriz, expresión de informaciones:

«en estas actividades de creatividad buscamos también mirar el desarrollo lógico... la organización de los juguetes por colores, forma, tamaño»

«el juego y el juguete, ... pueden ayudar en el niño a desarrollar sus habilidades motoras ya sean finas o gruesas»

«juego libre... Se puede trabajar conceptos, colores, cuantificadores, pero a ellos les encanta su juego libre».

En uno de los jardines se habla incluso de que en el juego los niños van a seguir con el tema de "la casa en el agua" que se venía trabajando.

De alguna forma, en todos los casos, aparece la función del juguete vinculada al trabajo más intencional del aula. Aunque todo parece indicar que este vínculo se da de manera espontánea ya que no existe una intervención del maestro para establecerlo. Así mismo, se afirma que hay materiales o juguetes, que posibilitan más este vínculo que otros: los rompecabezas, los bloques, los juegos de construcción, sin embargo estos últimos no fueron utilizados.

En el discurso del maestro, el vínculo entre el juguete, el juego libre y las actividades regulares de la clase, aparece como una de las funciones más importantes. Sin embargo, ¿por qué si se le atribuye este valor, en ninguna de las experiencias observadas se da una orientación o intervención en este sentido? Será acaso que el maestro cree que el juguete es útil para todo pero realmente no tiene una claridad sobre estas utilidades y quiere atribuírselas, en el discurso, para no sentir que se pierde el tiempo?

\section{El juguete como espacio para el desarrollo libre del niño}

Existe una valoración generalizada del juego libre como el espacio que permite el desarrollo del lenguaje, la creatividad, la imaginación.

«aquí hay diversos materiales para que los niños empiecen a desarrollar sus destrezas, no sólo motora sino también su creatividad... hay un espacio libre donde ellos exploran... es un espacio de juego libre»

«con las fichas es la imaginación de ellos, todo lo que quieran hacer» 
«el juego libre les permite... la alegría de ellos estar con sus juguetes, la diversión ... la expresividad de ellos que uno puede ver»

«en estas actividades observamos la creatividad... la idea acá es trabajar un poco la creatividad de los niños. Permitirles espacios donde ellos puedan desarrollarla».

Encontramos aquí el concepto de creatividad ligado al de libertad. Los niños crean cuando usan con libertad los juguetes, cuando pueden hacer lo que quieren. La idea parece afirmar que el juguete es algo del niño, que le da alegría, que propicia el uso de la imaginación y la creatividad, y en ese sentido podría justificarse la no intervención del maestro, pues equivaldría a entrometerse en el mundo "sagrado" del niño.

\section{El juguete como medio de expresión de la vida cotidiana del niño}

La utilización de juguetes como muñecas y objetos de la casa, incita a la realización de ciertos juegos en los cuales, para las maestras, el niño lo que muestra son las vivencias de su cotidianidad.

«Ellos juegan a lo que hacen en la casa, realmente uno se da cuenta por medio de sus juguetes a los que quieren o no los quieren según traten los juguetes o a veces ellos graban las peleas de los padres o de los hermanos y uno se da cuenta...»

«la idea de la profesión partió de ellos, y a partir de allí, cada uno, el que quiso integrarse a cada grupo escogió la profesión que más le gustaba»

«ellos expresan mucha alegría y al escucharlos ellos dicen muchas cosas que viven en la casa».
Esto último hace referencia al juego que en uno de los jardines se organizó sobre las pasadas elecciones presidenciales en las cuales los niños votaban por su candidato. Se habla, también, del teléfono como un juguete que permite "jugar a la vida misma".

Sin embargo, pareciera que el uso de los juguetes como expresión de la vida se convierte, a veces, en un test proyectivo: el niño muestra lo que vive en la casa, los conflictos, etc. Pero queda la duda de ¿qué pasa con la expresión de los conflictos de la casa?, ¿qué hace el maestro con eso?

En otros juegos (profesiones, casa) o en el juego de las elecciones, (organizado por los maestros), las maestras afirman que intervienen, tal vez porque se manifiestan las relaciones con el mundo de "lo adulto".

Sin embargo, el juego y el juguete no aparecen, en la visión del maestro, como un medio para reelaborar la vida cotidiana; apenas lo visualiza como un espejo de la vida.

\section{El juguete como reafirmador o integrador de las diferencias de género}

En el transcurso de las observaciones surge la inquietud, que se manifiesta explícitamente, sobre la interacción de niños y niñas. Aparece el uso de ciertos juguetes ligado al género.

«Algo muy marcado en las niñas, por lo general, son las muñecas, sus ollitas, en los niños son los carros o de construcción».
Aunque también se afirma,

«algunos niños que han sido agresivos... se ven jugando a la cocina, a las muñecas, se ven mucho más tranquilos, más tiernos, se ven como en otra faceta diferente».

0

«niños y niñas es heterogéneo, cuando hay una pelota ellos juegan al fútbol. Pero somos a veces los adultos los que les tenemos predestinado al niño que el juego del fútbol... es solamente para ellos, pero aquí tratamos de romper un poquito esa barrera»

«en este nivel se trabaja juego de roles... mira acá niños que hacen el almuerzo... que planchan... hay algunos que dicen usted hace el oficio y yo manejo el carro».

En los jardines privados parecería que se intenta relativizar las diferencias de juguete y género, lo cual no es muy claro en los públicos: sin embargo, aunque se vea como algo cultural, parece que ya está dado. No hay una intervención de las maestras en este sentido.

\section{El juguete como medio de socialización}

Para las maestras, el uso del juguete propicia las interacciones entre los niños. Por un lado, están las interacciones mismas, la reafirmación de las amistades o enemistades, la conformación de los grupos, los liderazgos; por otro, las intervenciones de la maestra sobre las anteriores.

Se hacen varias observaciones sobre los amigos, sobre la dificultad de integrar a algunos niños, de compartir: 
«la relación que desempeña cada uno, la colaboración, el compañerismo, si se integra fácil... buscamos líderes»

«pueden desarrollar así espacios de socialización, de tolerancia y respeto frente a ellos mismos»

«poco a poco los niños tímidos han tratado de relacionarse con los demás... en este momento el teléfono es importante... hacen que otro llame, que reciba el mensaje»

«yo observo mientras que juegan y luego hacemos una mesa redonda, y les voy preguntando qué hicieron y cómo lo hicieron y los niños más bruscos dan muchas quejas».

Aparece también el problema de los juguetes de cada niño en el jardín:

«en un tiempo... traían juguetes pero ... en esta edad no están dados a compartir con nadie, entonces se presentan muchos conflictos, peleas, que no me presta, que se me rompió, que ya no soy tu amigo, entonces decidimos no permitir que trajeran juguetes, además que muchos los traían a escondidas de la casa».

En los dos jardines privados se toma una decisión similar; los juguetes se dañan y ocasionan peleas. Si un niño quiere llevar un juguete "tiene que estar dispuesto a compartirlo". Aconsejan no llevar juguetes costosos ya que los padres forman problemas problemas si algo le pasa al juguete. Lo mejor es donarlos.

En uno de los jardines oficiales los padres llevan los juguetes nuevos al comienzo del año, y no se molestan si se dañan. «hay niños que tuvieron que llevarse su juguete para la casa porque no soportaron verlo aquí»

En general, en los jardines privados se opta por no aceptar el juguete individual; en el jardín, el juguete se vuelve colectivo.

En los jardines oficiales el juguete es individual, se marca con el nombre del niño, lo trae cada niño y es el que más le gusta. No es claro si el juguete sufre transformaciones en el jardín (¿se vuelve colectivo?).

Las entrevistas muestran también, que las maestras poseen un conocimiento sobre las amistades de los niños, sobre los grupos de amigos y el juego libre les permite confirmar estas interacciones. Pero la inquietud sigue siendo cómo potenciar estas relaciones: ¿la maestra debe intervenir o no?, ¿cómo?

\section{Papel del maestro}

Aunque las maestras no intervienen en el juego, este cumple un papel para ellas el que a su vez les da funciones como.

\section{Observar y analizar el comportamiento de los niños a través de lo que hacen}

El uso del juguete en el juego libre proporciona al maestro un conocimiento del niño; de ahí su papel de observador.

«el papel de nosotras por lo general es de observación... la creatividad... el desarrollo lógico-matemático... las relaciones que desempeña... si se integra fácil... buscamos líderes porque hay niños que motivan al grupo» «esto es básicamente lo que nos ayuda a entender mucho más al niño».

Si bien se adquiere mucho conocimiento observando el juego de los niños, cabe preguntarse: ¿desde dónde observa la maestra al niño?, ¿para qué le sirven ciertos tipos de observaciones y qué hace con ellas?, ¿para qué le sirve conocer los conflictos familiares?, ¿para qué le sirve conocer al niño que no presta los juguetes, para qué le sirve conocer al líder? estas preguntas surgen porque parecería que esas afirmaciones se pueden volver verdades estáticas "este niño es... la familia de este niño..." Queda la duda ¿qué pasa con la expresión de la vida y el comportamiento del niño?, ¿cuál es entonces el papel que el maestro se atribuye a este respecto?

\section{Orientar la recuperación de la actividad}

De alguna manera, pareciera que en algunos casos, las maestras busca una forma de intervención o recuperación de la actividad del juego libre, lo que se evidencia en los siguientes casos:

Una maestra pide a los niños que seleccionen los juguetes que quieran y luego les pregunta qué juguete seleccionaron: busca la expresión verbal de los niños. De igual forma, le atribuye algunas funciones cognitivas.

Otra maestra anuncia que al acabar la actividad se va a ver cuántos continuaron el tema de la casa en el agua, y se van a expresar los problemas y peleas que se 
dieron durante el juego. Pero al final, no se observa una acción de la maestra que permita recuperar la actividad ya que simplemente los niños cuentan lo que hicieron, pero la maestra no comenta nada.

Una de las maestras se refiere a la intervención que hicieron en un juego donde ellas también se disfrazaron, un juego de roles sobre las profesiones. Y la otra: cuenta que ella a veces juega con los niños ... y eso me permite relacionarme con ellos de una manera más afectiva... también retroalimenta los miedos.

Aunque las maestras intervienen en algunos casos, no es muy clara cómo debe ser esta intervención.

Otra duda que queda es si realmente la maestra está presente cuando se realiza esta actividad en el jardín, o si ella aprovecha este momento para ir a realizar otras obligaciones.

\section{Espacios donde se utiliza el juguete}

Además de las anteriores categorías, a raíz de las observaciones y la lectura de las entrevistas, se elaboraron otras dos, las referentes al espacio y tiempo para la utilización del juguete.

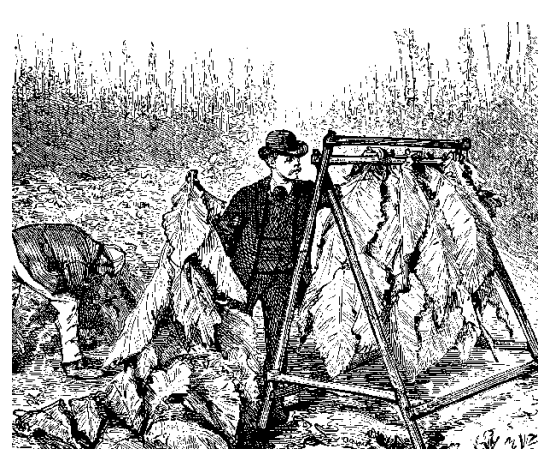

Las actividades de juguete planeadas se llevaron a cabo en diferentes espacios del jardín : en el primer jardín en un salón en donde había ya algunos juguetes (en este jardín cada grupo tiene su salón) ; en el segundo un salón en donde no había sino una colchoneta (aquí no hay salones por grupos sino por actividad : salón del cuento, del material de construcción, para pintar, etc.) ; En el tercero jardín, en el salón de los niños, allí están los juguetes, se inició la actividad, y se continúa en el parque, aunque aquí algunos juguetes pasan a segundo plano, ya que algunos niños se sintieron atraídos por el rodadero o el columpio ; En el último jardín, la actividad se realizó enel salón de clase de los niños.

Se observa que en los 2 jardines privados hay espacios especiales para el juego con juguetes o que pueden ser usados para ello. En los jardines oficiales es el salón de clase, aunque en uno fue utilizado también el parque. Seguramente las diferencias en las condicones locativas y la diferencia en el numero de niños que se atiende inciden en este aspecto, sin embargo no podemos olvidar que la organización espacial de la escuela implica un concepto de educación infantil, y en este caso de juego y juguete.

\section{Tiempo y actividad}

El tiempo dedicado a estas actividades es algo que inquieta en las diferentes observaciones. Al respecto las maestras plantean:

"en la semana por lo menos dos veces y por lo general al momento

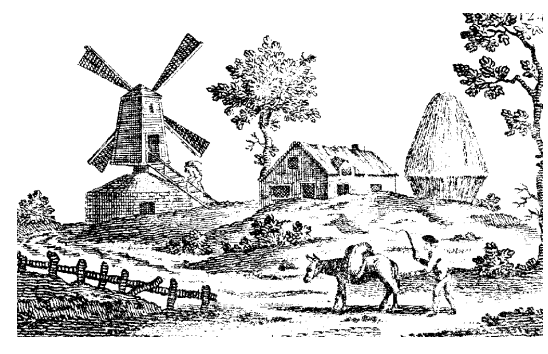

de la llegada se les hace este tipo de actividades.. la actividad dura máximo una hora, a partir de una hora ya empiezan a dispersarse, a hacer otras actividades"

"siempre tenemos diariamente
una actividad de juego, fuera de
otras actividades, siempre tene-
mos primordialmente juego... el
juego es una pirámide flotante,
juego, el cuento, y la parte de so-
ciabilidad dura aproximadamente
una hora... La actividad de juego
nunca se acaba, es al contrario,
uno les dice ya, gorditos, vamos a
organizar, a ellos se les rompe la
vida imaginativa de estos juegos...
nunca se cansan de jugar..."

"todos los días se juega, puede ser acá o en el parque, ellos disfrutan de los juguetes... tú no vez que ellos de pronto se aburran con sus juguetes, no, que ellos quieran cambiar de actividad, no... influye el espacio porque van a trabajar más rico allá (se refiere al parque)... ..puede ser toda una mañana... de nueve a once, normalmente... a nosotros nos corresponde de diez y media a once y cuarto de la mañana..."

"este tipo de actividades, casi todos los días con diferente materiales, plastilina...juguetes..., fichas..., más o menos como quince minutos, porque después se cansan y comienzan a pelearse".

Como se observa no es muy claro el lugar que ocupa el juego libre en el jardín. Las maestras tienden a decir que se realiza todos los días, lo que parece más 
cierto en los jardines privados, pero no parece tan claro en los dos oficiales (recordemos los juguetes en sus empaques). ¿Detrás de esta diferencia podríamos intuir que en estos dos tipos de jardines se encuentran diferentes valoraciones en cuanto a la utilidad misma del juego para el niño?, ¿los jardines privados lo considerarían más "útil" y los publicos más "pérdida de tiempo"?

En cuanto a la duración, la maestra del Jardín Privado 1 y la del Jardín Oficial 2 ven la actividad de juego libre como una actividad que se termina, que se agota; aunque para la primera se agota a la hora y para la segunda a los 15 minutos. Por el contrario para las otras 2 maestras la actividad nunca se terminaría. Queda la sensación de que las maestras creen que eso es lo que deben contestar en una indagación de este tipo: que la actividad de juego libre es muy importante para los niños, para el jardín y para ella.

\section{HALLAZGOS INICIALES}

Plateadas más como interrogantes, se presentan ahora algunas conclusiones, en las cuales sería necesario seguir profundizando, ya que el estudio mismo, cómo se ha dicho, es exploratorio sobre el tema.

Sobre el concepto de juguete:

1. Aunque en ningún jardín se hizo la pregunta directa, se encuentra una concepción explícita de juguete como "cualquier objeto que posibilite el juego".
2. El juguete es distinto del material didáctico; es algo específico que se utiliza preferiblemente en el juego libre. Sin embargo, aunque en el discurso las maestras afirman que sirve para apoyar otros aprendizajes, no se observa que las maestras lo utilicen como tal.

3. Existe la idea, aunque no desarrollada, de que diferentes tipos de juguetes sirven para diferentes logros. ¿Qué logros le atribuye la maestra a diferentes juguetes. Por qué en ninguna activiada esto es explícito?

4. De acuerdo a lo anterior, ¿en los jardines hay juguetes solamente para que los niños juegen libremente, sin ninguna organización ni intervención de la maestra?

Sobre la función del juguete y el papel del maestro:

1. El juguete como tal, tendería a cumplir su principal función en el juego libre; con éste el niño desarrolla libremente su creatividad y su imaginación.

2. El juego libre es el espacio "del niño", donde él se desarrolla, expresa su vida cotidiana, reafirma su género, se relaciona con los otros niños y en él, el maestro no debe entrar, no debe intervenir. En este sentido, ¿no habría diferencia entre el juego que el niño realiza en la casa y el que realiza en el jardín?

3. El papel del maestro es fundamentalmente de observador del juego libre; en él el maestro conoce la creatividad y la imaginación del niño, así como sus formas de relacio- narse con los demás niños y los conflictos de su vida cotidiana. ¿Qué hace el maestro con estas observaciones?, ¿son los juegos infantiles, como lo piensan las maestras, simples espejos de las situaciones familiares y no existen reelaboraciones de los niños? ¿Esto justifica la no intervención de la maestra? Las maestras intervienen en algunos juegos, cuando la vida "adulta en general" está presente, como en los juegos de oficios, de construcción, en juegos más sociales, donde ellas sienten que puede aportar, mas no en la vida íntima del niño.

4. El maestro no interviene en el juego porque considera que este es el "espacio sagrado" del niño. En este sentido, ¿La maestra no interviene por respeto a la intimidad del niño o porque no tiene elementos pedagógicos para intervenir?

5. El juego libre con juguetes le permite al niño continuar los aprendizajes escolares, pero de una forma espontánea; las maestras parecen saber que jugar con los juguetes les genera desarrollos a los niños, pero no es claro cómo se da esto y por qué.

Sobre el espacio y el tiempo del juego libre:

1. Hay espacios donde se favorece el juego libre: salones especiales, parque, etc. Pero aunque no siempre existan estos para las maestras es importante que los niños tengan espacios y tiempos para juegar. 
2. Aunque todas las maestras afirman que a los niños les fascina jugar, esto no se da ilimitadamente; ellas observan que después de algún tiempo, los niños buscan otro tipo de actividades. Para otras maestras, los niños permanecerían todo el tiempo en la actividad de juego libre, otras actividades les distraerían de su mayor placer. En este sentido, ¿que relaciones habría entre juego libre y otras actividades?

Sobre la diferencia entre el uso del juguete en el jardín privado y en el jardín público:

1. Función del juguete y papel del maestro: no se encuentran grandes diferencias entre los jardines privados y los jardines públicos en cuanto al uso del juguete y la función de la maestra ya que en las dos tipos de instituciones, en general, la actividad fue la misma, lo que podría indicar concepciones similares.

2. Espacios y tiempos: en los jardines privados son más definidos; los espacios para el juego y el juguete no necesariamente tienen que ver con el salón o con las actividades regulares. En los públicos, son indefinidos; igual puede ser el salón o el parque, no existen espacios específicos para el juego. ¿Implicara esto diferentes concepciones sobre la utilidad del del juego y del juguete en los niños?

3. Propiedad de los juguetes: en los jardines privados, los juguetes son del jardín, adquiridos por éste o donados por los niños. El juguete es colec- tivo, es de todos. En los oficiales, son de los niños, quienes traen sus juguetes preferidos. Aunque los juguetes estaban marcados cada niño podía escoger un juguete parecería que el juguete individual se vuelve colectivo. Sin embargo los juguetes de los jardines privados estaban más dañados, parecería que allí no era tan importante la calidad del juguete. ¿Lo anterior estara realcionado con el número y calidad de los juguetes que poseen los niños en su casa?

Como ya lo decíamos, estas afirmaciones y cuestionamientos se plantean más como hipótesis para estudios que profundicen, que como conclusiones generalizables. Pero el hecho de que todas las maestras al pedido de "realizar una actividad con juguetes" hubieran optado por una actividad de "juego libre" no deja de llamar la atención, como ya lo decíamos, existiría una idea fuerte que los juguetes son del "mundo de los niños" donde los adultos no debemos entrar porque eso coartaría su libertad su creatividad, en este sentido, al juego libre de por sí, se le atribuyen aprendizajes que se darían de forma espontánea.

Un custionamiento que surge es qué papel juegan las instituciones orientadoras del trabajo pedagógico infantil (La capacitación que imparte el Ministerio o el ICBF, la formación de las mismas universidades y centros de formación de maestras preescolares) en esta concepción de juego libre y uso del juguete. Convendría pensar programas de cualificación de maestros en lo cuales, sin negar el juego libre en si mismo es valioso, todas las actividades con juguetes no tendrían por qué ser tan espontáneas. Tal vez la organización de diversos espacios con juguetes pueden potenciar Juegos Libres más enriquecidos. Así mismo, adecuadas interveciones de las maestras, enriquecen el juego libre de los niños, como lo vemos en varias propuestas pedagógicas.

Sin embargo, estas capacitaciones no pueden olvidar la importancia de trabajar sobre las atribuciones que el maestro le da tanto al juguete como a la actividad. Al fin y al cabo se trata de comprender el pensamiento de las maestra, de sus porqués, para poder hacer un trabajo desde allí, valorando y poniendo en crisis estos pensamientos con el fin de cualificar este elemento fundamental de la educación infantil.

\section{BIBLIOGRAFÍA}

García, Marcelo. El pensamiento del profesor. CEAC, Barcelona, 1987.

GLANZER, Martha. "El rol estimulante de un buen juguete en el desarrollo del niño". En Un jardín de infantes mejor. Siete propuestas. Paidós, Buenos Aires, 1992.

MARTínez, M. La investigación cualitativa etnográfica en educación. Caracas: 1991.

Pérez, Ángel y Gimeno José. "Pensamiento y acción en el profesor: de los estudios sobre la planificación al pensamiento práctico". En Revista Infancia y aprendizaje, vol. 42, Barcelona, 1987. 


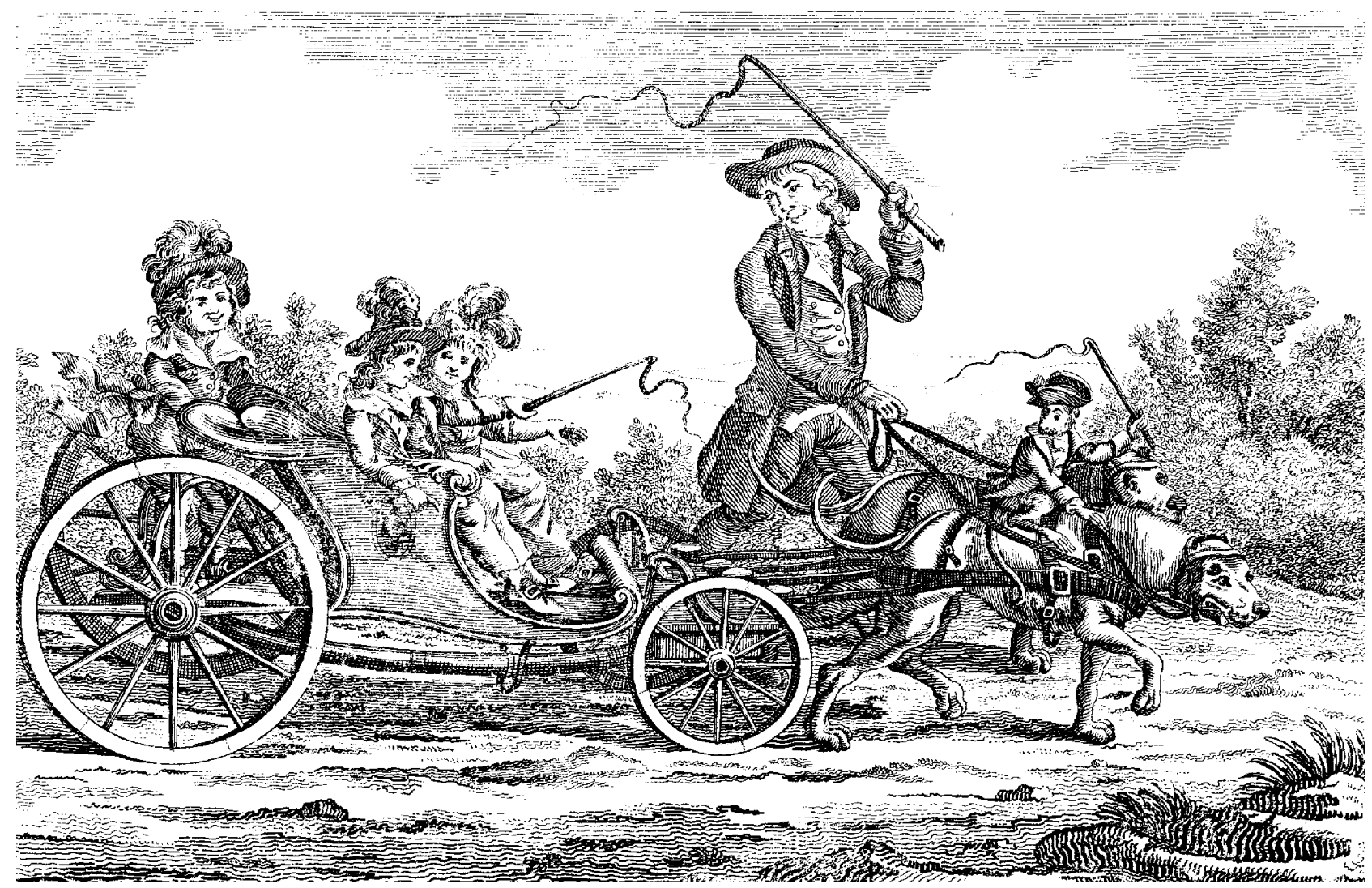

